\title{
LOS TEJIDOS ANDINOS, LA PASIÓN DE ELAYNE ZORN (1952-2010)
}

\author{
Cristina Bubba Zamora ${ }^{1}$
}

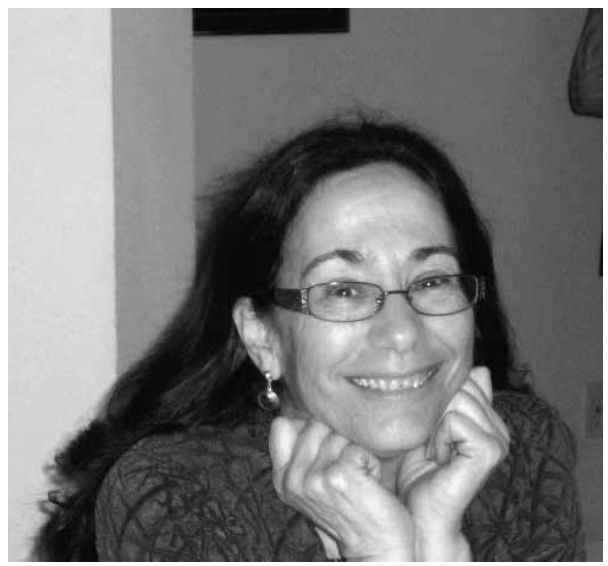

Elayne Zorn falleció el 15 de junio de 2010 en una batalla contra un cáncer de piel, que la afectó desde hace unos siete años. La conocí en los años 80 en una de sus visitas a Bolivia y nuestra amistad creció con el tiempo al igual que el apoyo profesional mutuo por el interés común en pueblos indígenas, en los tejidos y su significado.

En 1983 obtuvo un M.A. en la Universidad de Texas-Austin, en el departamento de Estudios Latinoamericanos. En 1987 logró otro M.A. en Antropología, en la Universidad de Cornell, Universidad en la que además obtuvo un Ph.D., con una interesante disertación: Marketing Diversity: Global Transformation in Cloth and Identity in Highland Peru and Bolivia.

Entre 1991 y 1992 fue investigadora asociada en la Universidad de Liverpool y el Kings College, Londres. Luego trabajó como asistente enseñando antropología y estudios de mujeres, en la Universidad de Cornell entre 1985-1986 y 1992-1994.

En Colgate University, entre 1994 y 1998, enseñó antropología y escritura interdisciplinaria. Desde 1998 hasta el presente fue profesora asistente en antropología, luego profesora asociada y finalmente directora asociada del Laboratorio de Etnología Digital, en la University of Central Florida.
Realizó trabajos de campo en diferentes lugares de los Andes, entre los importantes se pueden citar los llevados a cabo en Taquile, Perú, en varias oportunidades: 1975-76, 1978, 1980-81, donde centró sus investigaciones en textiles, género, turismo y aprendió a tejer como una tejedora local. También visitó Puno estudiando artesanías y su comercialización en varias oportunidades.

Voy a referirme a tres artículos sobre tejidos, de los muchos que escribió Elayne, como resultado de sus estudios llevados a cabo en diferentes lugares de los Andes y que se constituyen en un invalorable aporte para el conocimiento y comprensión del textil, desde el punto de vista de la identidad, la tradición y su uso actual.

Uno de los que más me llaman la atención es: Reading Cloth: "The Text" in Textiles ${ }^{1}$, en el que nos cuenta como los Taquile escriben en sus llamadas fajas calendario los meses del año con doce dibujos, acompañados por dos páginas escritas a mano, por los esposos de las tejedoras, que explican los mismos. Son llamadas: wata yapananpaq chumpi, faja para contar los meses del año.

Los diseños son diferentes y son colocados en secuencia, a diferencia de lo que ocurre en las fajas tejidas para uso cotidiano. Elayne consideraba que

Fundación Illa (Saberes y Memorias), Casilla 14066, La Paz, Bolivia, c.bubba@megalink.com 
estos nuevos diseños y secuencias cambiaron para simplificar y lograr que la faja sea más inteligible a los occidentales y recientemente producidas en respuesta a la demanda de los compradores. Estas fajas no tienen terminaciones para asegurar la misma alrededor de la cintura de la persona que la usa, sino un palito y un hilo colocado para que el mismo sea colgado en una pared.

Una de las tejedoras de estas prendas, Agustina Huatta, contó a Elayne que como no escriben en quechua, tejen para poder "recordar". Pero estas nuevas fajas, a diferencia del resto de los textiles que producen los Taquile para vender, no son comercializadas en la tienda comunal del pueblo, se las podía adquirir solamente en la casa de las tejedoras.

En Bolivia se concentró en el estudio de los tejidos del norte de Potosí, en los del ayllu de Sakaka, que fueron parte de la Federación preinca Charca, ubicados en la provincia Alonso de Ibáñez, departamento de Potosí, donde llevó a cabo su trabajo de campo para su tesis doctoral entre los años 1986 y 1988.

Los Sakaka, según Zorn, son unos apasionados por los tejidos y bordados ${ }^{2}$. El textil y la ropa en este grupo étnico cumplen importantes funciones y tienen valor de representación, algunos de ellos son:

(1) El extremo o supremo signo de representación y construcción de la identidad en sus múltiples formas.

(2) Son un sistema de comunicación, con significados y valores que los textiles, tomados en su conjunto, expresan y constituyen para las tejedoras.

(3) La más importante expresión visual de los habitantes de los Andes.

(4) La ropa tiene una función poética para las tejedoras y el resto de los miembros de su comunidad.

(5) La ropa tiene una significativa función económica, que incluye bienestar, ya sea expuesta al público, guardada en casas o heredada.

A partir del análisis de sus funciones y representaciones, y al constituirse en un supremo signo de construcción de la identidad en sus múltiples formas, individual y colectiva, de grupo étnico, de región y otros, permiten al observador saber con precisión la identidad del que los usa, su afiliación étnica, su estatus económico y social. Son pues el más importante elemento de expresión de las identidades en las culturas de los Andes. De ahí la importancia de la clasificación que lleva a cabo Elayne de los diferentes estilos que nos mostrará el lugar que ocupa la persona que los usa en la sociedad de los Sakaka, o al que pretende llegar a ocupar cambiando ropa de un estilo o a otro, marcando así su posición social y su nivel económico. Los estilos están relacionados con categorías sociales como los runa, "cholo o cholita"3, los mestizos y mestizas y los q'ara o blancos.

Dentro de los estilos utilizados por los runa, está el llamado clásico estilo tradicional, ñawpa muda runa p'ach $a^{4} \mathrm{o}$ antigua moda de ropa de runa, es utilizado por los runa (como se llaman a sí mismos los Sakaka, que en quechua quiere decir gente), en la medida en que los diferencia de los q'ara (no "indios", desde el punto de vista de los runa) que utilizan ropa al estilo occidental y de los "cholos o cholitas". Los Sakaka que visten este estilo viven en regiones altas y frías.

Estas prendas son enteramente elaboradas a mano por ellos. Están tejidas con lanas de alpaca, llama y oveja hilada a mano, por lo general de sus propios rebaños, teñida con tintes naturales y tejidas en telares tradicionales. Las mujeres visten los ajsu (medio vestido), awayu o llijlla (manta), almillas (vestidos) fabricadas con bayeta de la tierra y bordadas, chumpi (cinturón o faja), y llevan unkhuña (tejidos pequeños de una sola pieza para llevar hojas de coca, merienda y otros). Los hombres visten poncho, pantalones, chalecos bordados y sacos elaborados con bayeta de la tierra, gorros o ch'ulu tejidos a palillo, y llevan ch'uspa (bolsa) para guardar hojas de coca. Ambos sexos usan sombreros blancos y abarcas confeccionadas con goma de llantas de autos, ambas prendas son fabricadas fuera del ayllu. Este estilo es utilizado por gente de mediana edad y mayores, adolescentes pobres e hijos de familias con tradición en tejer vestimenta.

El estilo moderno tradicional llamado musuj muda runa p'acha que puede traducirse como moda nueva de ropa de runa, es utilizada por adolescentes y gente de mediana edad, que tienen más ingresos económicos, pequeños montos de dinero en efectivo por los trabajos temporales que llevan a cabo fuera del ayllu, como agricultores o en el Chapare y tienen además más conciencia de la moda.

Se utilizan las mismas prendas, pero nuevos diseños son tejidos en los awayu y otros, como por ejemplo helicópteros y motocicletas. Se introduce una estética nueva y se utilizan diferentes técnicas textiles. Los bordados en las almillas y los chalecos son más recargados, que los del estilo anterior. Por lo general, se utiliza lana sintética, comprada en los mercados locales, que son hiladas y tejidas a mano. Los colores son diferentes a los del estilo tradicional, más verde limón que reemplaza al blanco muy utilizado en el estilo anterior. 
Un tercer subestilo de esta categoría es el que implica el uso de algunas prendas de ropa fabricada fuera del ayllu y comprada; por ejemplo, las mujeres utilizan vestidos fabricados con telas sintéticas y faldas, que no son tradicionales como los usados en los estilos anteriores. Mientras que los hombres usan chalecos, chaquetas y chalinas compradas. Que son producidas a máquina por miembros del ayllu Laymi en el norte de Potosí. Mientras otras prendas como el poncho, las cintas tejidas utilizadas alrededor de los sombreros, los ch'ullu (gorros) y algunos awayu siguen siendo tejidos en la comunidad y son los que todavía están marcando la identidad étnica de los Sakaka.

Estas prendas compradas son todas producidas en un estilo diferente que Zorn llama norte de Potosí, es significativo ya que quizás los jóvenes Sakaka al cambiar su estilo de ropa están perdiendo la identidad Sakaka y la relación con su ayllu de origen para integrar un grupo más amplio formado por los varios pueblos indígenas que habitan el norte de Potosí, que será la nueva identidad étnica en un futuro no muy lejano.

Una variación de este estilo es descrita como aquella en la que los Sakaka adquieren las prendas mencionadas anteriormente de los Laymi, pero sin dibujos y bordados, los que son llevados a cabo por ellos mismo el estilo norte de Potosí o Sakaka.

El cambio de un estilo de vestimenta a otro representa para los Sakaka un cambio de estatus social y económico.

La ropa utilizada por los "cholitos o cholitas" que viven en los pequeños pueblos y han dejado de vestirse de manera tradicional es fabricada enteramente con fibras sintéticas. Las mujeres visten polleras, sombreros y mantas compradas, mientras que los hombres se visten más al estilo occidental con pantalón, saco y por lo general llevan sombrero negro y no blanco como en las anteriores categorías. En el estilo cholita p'acha, ropa de cholita, se combina con por lo menos un elemento o prenda que tenga relación con la identidad de los Sakaka, es decir, el awayu, tejido en telar tradicional o la faja tejida utilizada en el sombrero, ambas prendas con diseños característicos que los identifican como Sakaka, aunque estén vestidos de cholitos o cholitas.

Para terminar se refiere a la categoría $q$ 'ara p'acha o civil p'acha que es la ropa de los blancos o civiles que es enteramente fabricada al estilo occidental, que no es utilizada por los Sakaka.

Este resumen de las interesantes descripciones y observaciones que llevó a cabo Zorn, es un ejemplo de como el análisis de la ropa y sus modificaciones puede dar indicios de los cambios en las estructuras sociales de grupos étnicos, como en este caso los Sakaka y los cambios que se están produciendo a nivel de su identidades, aspiraciones sociales y relaciones con otros grupos o clases sociales.

Su trabajo no se limitó a lo científico y las publicaciones, sino que también tomó partido por las luchas y acciones de los pueblos indígenas de Perú y Bolivia, es así que cuando los tejidos de Coroma fueron incautados en San Francisco, California, Estados Unidos, Elayne apoyó nuestro proceso con un grupo de profesionales norteamericanos comprometidos con los Andes y logró la firma de importantes votos resolutivos a favor de Coroma y nuestra causa, por lo que fue amedrentada y amenazada por los traficantes. Apoyó también con su conocimiento para que un tejido de Coroma que estaba en un Museo en los Estados Unidos fuera devuelto a los ayllu de origen en el año 1996.

Dos cosas se asemejan en nuestras investigaciones y estudios, sin saberlo ambas nos topamos con q'ipi o atados, ella en Mascusani, capital de la provincia Carabaya, en Puno, Perú, donde las familias conservan señal q'ipi (atados) para la realización de rituales en los que sus animales son marcados y yo con los $q^{\prime}$ 'ipi sagrados ${ }^{5}$ de los ayllu de Coroma, ubicados en la provincia Quijarro del departamento de Potosí, en Bolivia. Cuando necesito alguna referencia para comparación la única que tengo es la que Elayne nos dejó. Una diferencia importante está marcada por el tipo de propiedad, los tejidos ceremoniales de los q'ipi de Coroma son propiedad colectiva de los miembros de cada ayllu, mientras que los de Mascusani pertenecen a cada familia.

De acuerdo con esta investigadora, los señal $q^{\prime} i p i^{6}$ están compuestos de una serie de elementos rituales, que son utilizados para las ceremonias del señalakuy, es la ceremonia más importante para los pastores de Puno, que se realiza dos veces al año, en febrero y agosto, para marcar o señalar a sus animales (alpacas, llamas, ovejas y otros) y para pagar a la Pachamama y a los Apu (espíritus de los cerros). Ceremonias de mucha importancia para los pastores de Puna, ya que sin animales y pastoreo no hay vida a esa altura.

Es realizada por las familias de pastores y tiene una duración de por lo menos una noche y un día; puede extenderse hasta una semana. En este ritual, que tiene una serie de ceremonias en sí, como describe Elayne, desde el sacrificio de una llama blanca 
hasta la apertura del señal $q$ 'ipi, acompañadas de las canciones de las mujeres a los animales y finalmente el corte de una pequeña parte de las orejas a cada tipo de animales, en la que cada familia pone su propia señal a sus rebaños.

Los tejidos juegan un rol preponderante en estos rituales. Los pastores guardan figuras en miniatura que representan la fertilidad de cada tipo de animal en wayaqa (bolsas) separadas dentro del señal q'ipi. Además, cada unkhuña, al interior del q'ipi, conserva por lo menos la figura de un animal, la comida cruda que necesita, más su pasto y hojas de coca.

El señal $q^{\prime}$ ipi es llamado mama q'ipi ${ }^{7}$, es decir, madre atado. El bulto es la madre de los pastores, su progenitora y protectora de la fertilidad. Cada uno de estos señal $q$ 'ipi contiene al interior otros q'ipi más pequeños. El textil que envuelve cada q'ipi es el q'ipiñ $a$, que en otras regiones es llamado awayu o llijlla, más una o más awasqa (soga) y por lo menos dos o más unkhuña. Algunos contienen wayaqa costales pequeños, ch'uspa (bolsa para llevar hojas de coca) y animal unkhuña.

Las mujeres de Mascusani emplean diferentes términos para referirse a las unkhuña. Las diferencian por tamaño, por color y organización del espacio, y finalmente por función, así por ejemplo la coca unkhuña, la comida unkhuña, la animal unkhuña: una para alpacas, otra para llamas, otra para ovejas y finalmente una para las vacas, y la misma unkhuña para preparar el ritual.

Zorn realiza además una interesante y minuciosa descripción de las técnicas utilizadas, los colores y la organización del espacio de este tipo de prendas, a partir de la cual lleva a cabo una clasificación partiendo del centro liso llamado pampa. En la tercera clasificación están las unkhuña que tienen una división dual de la pampa. La que está dividida en dos áreas iguales, por el contraste entre el encuentro de los dos colores que produce la sensación de una división en dos, estas son llamadas en Mascusani tijlla unkhuña ${ }^{8}$.

Es interesante que en el otro extremo del altiplano en los ayllu de Coroma, en las ch'alla o libaciones durante los rituales que llevan a cabo a sus q'ipi ceremoniales de tejidos, recuerdan a las almas fundadoras de estos ayllu, una de ellas llamada María Tijllama9 . Según el diccionario quechua de González Holguín, tijlla es definido como allca o ticlla. Allca es el contraste de dos colores, concepto estudiado por Cereceda (1978). Y ticlla tijlla en el mismo diccionario es "cosa hecha de los dos colores, blanco de vna haz y negro de otra como camiseta". De ahí quizás la relación que llevan a cabo los Coroma, en vista de que la mayoría de las prendas conservadas en los q'ipi son camisetas o qhawa nombre en aymara y recordando posiblemente una técnica de tejer muy antigua, usada en este tipo de prendas, mientras que en Mascusani se refieren con este término al contraste de dos colores mencionado en el caso de las unkhuña.

Elayne hablaba el quechua con más fluidez que el español y logró avanzar en sus estudios del aymara. En sus idas y venidas a los Andes, no solamente se enamoró de los pueblos indígenas, sus costumbres, música, tejidos y otros, sino que también de uno de sus miembros y se casó con Juan Cutipa Colque, músico de Puno.

La última vez que Elayne nos visitó fue en 2006, entre enero y agosto en su medio año sabático. Quiso retornar nuevamente pero no consiguió fondos. En esa ocasión estuvimos juntas en la posesión de Evo Morales en Tiwanaku, admirando la maravillosa vestimenta que lucían los representantes de los diferentes pueblos indígenas y originarios. Vimos por televisión la posesión en el Congreso y el hermoso saco que lució el Presidente en esa ocasión, que tenía un fragmento de textil como adorno y símbolo. Nos quedamos pensando si ese fragmento fue tejido para la ocasión o era un fragmento de un tejido quizás antiguo. Ante la preocupación de que tejidos antiguos e importantes sean destruidos, como sucede cotidianamente con el corte de tejidos para la confección de carteras, mochilas, chalecos y hasta zapatos. Escribimos una carta pidiéndole que por favor no utilice fragmentos de tejidos antiguos en su ropa, por el significado que tienen los tejidos en su conjunto y por ser considerados elementos vivos. Las leyes bolivianas prohíben su corte o destrucción. Le sugerimos que contraten tejedoras para tejer fragmentos, nunca tuvimos respuesta. Nuestro Presidente sigue utilizando trajes con fragmentos de tejidos.

Elayne pertenece a la generación de antropólogos que aman los Andes y realizan trabajos muy comprometidos con sus procesos a favor de los pueblos indígenas y originarios. Su aporte al conocimiento de los tejidos es fundamental, y me pregunto qué otros interesantes estudios hubiera llevado todavía a cabo.

Su hijo Gavriel la ha sobrevivido, su madre ha fallecido hace cuatro meses.

Querida Elayne, espero que estés como siempre sonriente, optimista y muy solidaria, así es como te voy a recordar.

La Paz, mayo de 2013 


\section{Referencias Citadas}

Bubba, C. 1993. Nos querían robar el alma. Cuarto Intermedio 29:34-64. Cochabamba.

- - - 1997. Los rituales a los vestidos de María Titiqhawa, Juana Palla y otros fundadores de los ayllu de Coroma. En Saberes y Memorias en los Andes. In Memorian Thierry Saignes, compilado por T. Bouysse-Cassagne, pp. 377-400. IHEAL/IFEA, Lima.

Cereceda, V. 1978. The semiology of Andean textiles: the talegas of Isluga. Antropological History of Andean Polities. London, Cambrigde University Press, Cambridge.

Gonzáles Holguín, D. 1989. Vocabvlario de la lengva general de todo el Perv llamada: lengua qquichua o del Inca. (1608). Universidad Mayor de San Marcos. Lima, Perú.
Zorn, E.1986. Textiles in Herder's Ritual Bundles in Macusani, Peru. En The Junius B. Bird Conference on Andean Textiles, editado por A.P. Rowe, pp. 289-307, The Textile Museum, Washington, D.C.

- - - 1992. Reading Cloth: "The Text" in Textiles. Disertación presentada en el Metodologies Seminar, Center for Latin American Cultural Studies, King's College London. May 19.

- - - 1999. (Re)Fashioning the Self: Dress, Economy, and Identity among the Sakaka of Northern Potosí, Bolivia. Chungara 30:161-196.

\section{Notas}

Ver Zorn, "Reading Cloth...” (1992).

Ver Zorn, “(Re)Fashioning the Self...” (1999).

Estos términos son usados por los Sakaka para definir este grupo social.

4 Agradezco a Xavier Albó por la corrección de la ortografía de las palabras en aymara y quechua.

5 Ver Bubba, "Nos querían robar ..." (1993).
6 Se ha llevado a cabo un resumen del artículo de Zorn, "Textiles in Herder's Ritual Bundles..." (1986).

7 Ver Zorn, “Textiles in Herder's Ritual Bundles...” (1986:291).

8 Ver Zorn, "Textiles in Herder's Ritual Bundles..." (1986:295).

9 Ver Bubba, "Los rituales a los vestidos de María Titiqhawa..." (1997:387).

\section{Bibliografía Zorn}

\section{Libros y monografías (con revisión de pares)}

Underberg, N. y E. Zorn, Digital Ethnography. University of Texas Press, Austin, ms en revisión.

Zorn, E. 2004.Weaving a future: Tourism, cloth, and culture on an Andean Island.University of Iowa Press, Iowa City.

Artículos (con revisión de pares)

Chase, A.F., D.Z. Chase, E. Zorn y W. Teeter 2008. Textiles in the Maya Archaeological record: Gender, power, and status in Classic Period Caracol, Belize. Ancient Mesoamerica 19:127-142.

Zorn, E. y L. Clare 2007. Fart farthing, communitarian tourism: Hosts and mediators in Peru. Annals of Tourism Research 34:673-689.

Ypeij, A. y E. Zorn 2007.Taquile: A Peruvian tourist island struggling for control. European Review of Latin American and Caribbean Studies 82:119-128.

Zorn, E. 1999. (Re)Fashioning the self: Dress, economy, and identity among the Sakaka of Northern Potosi, Bolivia. Chungara 30:161-196.

Zorn, E. 1987. Un análisis de los tejidos en los atados rituales de los pastores. Revista Andina 5(2):489-526.

Healy, K. y E. Zorn 1982-83. Lake Titicaca's Campesino-controlled Tourism. Grassroots Development 6(2)/7(1):3-10.

\section{Reimpreso en:}

Cultural Expression and Grassroots Development: Cases from Latin America and the Caribbean, editado por Ch.D. Kleymeyer, pp. 135-148. Lynne Rienner Publishers Boulder 1994.
La Expresión Cultural y el Desarrollo de Base, editado por Ch. Kleymeyer, pp. 207-224.Washington, D.C./Quito: Inter-American Foundation/Ed. Abya-Yala, 1993.

Direct to the Poor: Grassroots Development in Latin American, editado por S.Annisy P. Hakim, pp. 45-56. Lynne Rienner Publishing, Boulder 1988.

Zorn, E. 1982 [1980-81]. Sling braiding in the Macusani Area of Peru. Textile Museum Journal 19-20:41-54.

Seligmann, L.J. y E. Zorn 1981. Visión diacrónica de la economía de la producción textil andina. América Indígena XLI(2):265-287.

Zorn, E. y L.S. Lieberman Ms. Culture and Nutrition of the Andean, Potato in a Globalizing World. In Globalizing Food in the Americas: Anthropological Perspectives on the Political Economy, Forms of Resistance and Negotiation of Identities through Food, editado por L. Stanford, Berg, New York. (Accepted 9/06).

\section{Capítulos de libros (con revisión de pares)}

Zorn, E. y L.S. Lieberman 2007. Globalization of the potato: An ancient Andean indigenous crop. En Sustainable Development: Issues and Perspectives, editado por R. N. Patiy Odile S. SchwarzHerion, pp. 359-371. New Delhi: D.K. Printworld (P) Ltd., with support from IK Foundation of India.

Zorn, E. y L. Farthing 2006. Desafíos de un turismo controlado por la comunidad: el caso de la Isla Taquile, Perú. En La Ruta Andina. Turismo y Desarrollo Sostenible en Perú y Bolivia, editado por A. Ypeij y A. Zoomers con J. Gómez, pp. 61-84. Quito/Lima/Cusco/Amsterdam: AbyaYala/Instituto de Estudios Peruanos/Centro Bartolomé de las Casas/CEDLA. 
Zorn, E. 2005. Dressed to kill: The embroidered fashion industry of the ritual battle fighters of Highland Bolivia. En Latin American Fashion, editado por R. Root, pp. 114-141. Berg Publishers, Oxford.

Zorn, E. 2005. From political prison to tourist village: Tourism, gender, indigeneity, and the Nation in Taquile Island, Peru. En Natives Making Nation: Indigeneity, Gender and the State in the Andes, editado por A. Canessa, pp.156-180. University of Arizona Press, Tucson.

Zorn, E. 2002. Dangerous encounters: Ritual battle battles in Andean Bolivia. En Combat, Ritual, and Performance: Anthropology of the Martial Arts, editado por D.E. Jones, pp. 119-152. Praeger, Westport.

Zorn, E. 2000. When Incas travel abroad: Tourism to and from Peru. En Transforming Cultures in the Americas, editado por D. Castillo y M.J. Dudley, pp. 21-30. Vol. 4. Latin American Studies Program/Cornell University, Ithaca.

Zorn, E. 1997. Coca, cash, and cloth in Highland Bolivia: The Chapare and transformations in a traditional Andean textile economy. En Coca, Cocaine, and the Bolivian Reality, editado por M.B. Léonsy H. Sanabria, pp. 71-98. State University of New York Press, Albany.

Zorn, E. 1987. Encircling Meaning: Economics and Aesthetics in Taquile, Peru. En Andean Aesthetics: Textiles of Peru and Bolivia, editado por B. Femenías, pp.67-80. Elvehjem Museum of Art, University of Wisconsin, Madison.

Zorn, E.1986. Textiles in herder's ritual bundles in Macusani, Peru. En The Junius B. Bird Conference on Andean Textiles, editado por A.P. Rowe, pp. 289-307.The Textile Museum, Washington, D.C.

Zorn, E. Ms. Cambios en la producción y uso de los tejidos en Taquile, Perú. En Runakunap kawsayninkupaq rurasqankunaqa. La Tecnología en el Mundo Andino, editado por H. Lechtmany A.M.Soldi,Vol. 2. Universidad Autónoma de México, México City. (Accepted 8/01).

\section{Trabajos Enciclopédicos}

Everett, D. y E. Zorn 2008. Encyclopedia of Native American Artists Westport, C CT: Greenwood Press.

Cahlander, A., E. Zorn y A.P. Rowe 1980. Sling braiding of the Andes, Weaver's Journal Monograph IV. Colorado Fiber Center, Boulder.

\section{Artículos en Trabajos Enciclopédicos}

Zorn, E. 2010. Evo Morales and the Politics of Dress in Bolivia. The Encyclopedia of World Dress and Fashion, editado por M. Schevill y B. Femenias, Berg Publishers, Oxford (Forthcoming May 2010), en prensa.

Zorn, E. 2010. The cloth of the Sakaka of Bolivia, The Encyclopedia of World Dress and Fashion, editado por M. Schevill y B. Femenias, Berg Publishers, Oxford. (Forthcoming May 2010), en prensa.

Zorn, E. 2010. The Textile arts of Taquile Island, Peru. The Encyclopedia of World Dress and Fashion. M. Schevill y B. Femenias, Berg Publishers, Oxford. (Forthcoming May 2010), en prensa.
Publicaciones de ponencias en congresos (con revisión de pares)

Zorn, E. 2007. Weaving messages today: Three decades of belts in Taquile Island, Peru (1976-2006). En Textile narratives + conversations. Proceedings of the 10th Biennial Symposium of the Textile Society of America, October 11-14, 2006, Toronto. Pp. 1-10. CD-Rom. Textile Society of America, Toronto.

Zorn. E. 2005. Transformations in tapestry in the Ayacucho Region of Peru. En Appropriation, Acculturation, Transformation. Proceedings of the Ninth Biennial Symposium of the Textile Society of America, October 6-9, 2004. 10 pp. CD-Rom. Textile Society of America, Toronto.

Zorn, E. y L.S. Lieberman 2004. Freeze-dried but always peeled: Anthropological approaches to food processing, preparation, and consumption of the Andean potato. En Proceedings of the Conference on Indigenous Knowledges: Transforming the Academy, ICIK, University Park: The Pennsylvania State University. http://www.ed.psu.edu/ICIK/2004Proceedings/ section2-zorn-lieberman-withpics.pdf 14 pp. (July 2004).

Zorn, E. y J. Quispe 2004. The cultural significance of Andean clothand implications of its decline. En Proceedings of the Conference on Indigenous Knowledges: Transforming the Academy, ICIK, The Pennsylvania State University, Park. http:// www.ed.psu.edu/icik/2004Proceedings/section5-zorn-quispewithpics.pdf, 9 pp. (July 2004).

Zorn, E. 1995. (Re-) Fashioning identity: Late Twentieth-Century transformations in Dress and Society in Bolivia. En Contact, Crossover, Continuity. Proceedings of the Fourth Biennial Symposium of the Textile Society of America, September 22-24, 1994. Pp. 343-354.Textile Society of America, Toronto.

Zorn, E. 1993.Textiles as a daily obsession in the Andes. A day in the life of an Andean weaver. En Textiles in Daily Life. Proceedings of the Third Biennial Symposium of the Textile Society of America, September 24-26,1992. Pp. 151-162. Textile Society of America, Washington, D.C.

Zorn, E. 1991. Modern traditions: The impact of the trade in traditional textiles on the Sakaka of Northern Potosi, Bolivia. En Textiles in Trade. Proceedings of the Second Biennial Symposium of the Textile Society of America, September 14-16, 1990. Pp. 241-252. Textile Society of America, Los Angeles.

Zorn, E. 1979.Warping and weaving on a four-Stake Ground Loom in the Lake Titicaca basin community of Taquile, Peru, En Looms and Their Products: Irene Emery Roundtable on Museum Textiles, 1977 Proceeding Proceedings, editado por I. Emery y P. Fiske, pp. 212-227. Textile Museum, Washington D.C.

\section{Reseñas}

Zorn, E. 1999. Review of The contemporary tapestries of Alejandrina Ayme de Jiménez, Exhibition at the Clough-Hanson gallery, Rhodes College, Memphis. The Textile Society of America Newsletter 11(2):9-10.

Zorn, E. y A. Peters 1997. Review of Carol Hendrickson, weaving identities: Construction of dress and self in a Highland Guatemala Town. The Bulletin of Latin American Research 16(2):233-234.

Breves contribuciones (comentarios y prensa)

Cutipa, J. y E. Zorn 1990. 24 Hours in the Life of an Andean Herder. Llamas 4(2):97-100 (March/April). 
Cutipa, J. y E. Zorn 1985. The dances of Puno, Peru, Faces (February):30-33.

Healy, K.y E. Zorn 1983.Taquile's Homespun Tourism. Natural History 92 (11): 80-93.

Zorn, E. y N. Underberg 2009. Multisensory immersion in virtual spaces: PeruVine/PeruDigital. Anthropology News, pp. 18-19 (April).

Zorn, E. 1994.Taquile, Peru: Model T Tourism 1994 Festival of American Folklife (Catalogue), P. 22, Smithsonian Institution, Washington D.C.

Zorn, E. y J. Cutipa 1985. A closer look at alpacas. Spin-Off $9(2): 21-23$.

Zorn, E. 1981.Textiles and tourism: The shift to small commodity production in the Peruvian Highlands. Andean Perspectives Newsletter 3:11-13.

Zorn, E. 1978. Tejidos de Taquile. Taquilekunaqawasqan (Textiles of Taquile). (Curatorial Guide). Puno: Concejo Provincial de Puno, Perú. Mimeografeado. N.p. (Printed separately).

\section{Informes}

Zorn, E. y A. Ugarte 1978. Agricultural cooperatives in the Chapare region of Bolivia, The Inter-American Foundation, W Washington, D.C.

\section{Trabajos en Medios de Comunicación}

2007-present Zorn, E, y N. Underbergy. El PeruVine/PeruDigital Team, Co-, direction and development of the multilingual (English and Spanish) website PeruVine/PeruDigital, Digital Ethnography Lab, University of Central Florida http://www. peruvine.org orhttp://www.perudigital.org. Quechua version in preparation for Fall 2009.

2006 Zorn, E. y el Folkvine Team, Nicario Jiménez website, Folkvine.org,Cultural Heritage Alliance, University ofCentral Florida http://www.folkvine.org. Also assisted withwebsites of Eileen Brautman and the Lacemakers.

1992 Howard-Malverde, R. y E. Zorn, Kawsay Vida. Life and Language in the Andes, King', King's College London and the University of Liverpool. PAL laser videodisc and integrated video system, HyperCard interactive computer program.

1990 Peters, A. y E. Zorn, Andean Textiles, in The Andean World C. Brod \& Latin American Studies Program, producers. Intellimation \& Cornell University. HyperCard interactive computer program.

1985, 1994 Cutipa, J.y E. Zorn, Flutes and Strings of the Andes: Native Musicians from the Altiplano, Music of the World. Audio cassette MOW 106. Recorded music and provided notes.

1977 Zorn, E. Achayta noqayku ruwayku (That's Our Way). Video tape presented at Loom and Their Products: Irene Emery Roundtable on Museum Textiles. The Textile Museum, Washington, D.C. 
